\title{
Clinical significance of locoregional and systemic treatment in operable high-risk breast cancer patients with more than four positive axillary lymph nodes
}

This article was published in the following Dove Press journal:

OncoTargets and Therapy

2I September 2015

Number of times this article has been viewed

Kai Yin'

Liheng Zhou ${ }^{2}$

Zhimin Shao'

Wenjin Yin'

Jinsong $\mathrm{Lu}^{2}$

'Department of Breast Surgery, Fudan University Shanghai Cancer Center; Department of Oncology, Shanghai Medical College, Fudan University, Shanghai, People's Republic of China; ${ }^{2}$ Breast Cancer Center, Renji Hospital, School of Medicine, Shanghai Jiaotong University, Shanghai, People's Republic of China

Correspondence: Wenjin Yin Department of Breast Surgery, Fudan University Shanghai Cancer Center; Department of Oncology, Shanghai Medical College, Fudan University, 270 Dongan Road, Xuhui, Shanghai 200032, People's Republic of China Email followroad@I63.com

Jinsong Lu

Breast Cancer Center, Renji Hospital, School of Medicine, 160 Pujian Road, Pudong, Shanghai Jiaotong University, $200 I 27$ Shanghai, People's Republic of China

Email: lujjss@I63.com
Purpose: Breast cancer cases with four or more involved axillary lymph nodes (ALNs) feature an aggressive clinical history despite intensive treatment. However, therapies for improving the prognosis for these high-risk patients and the prognostic role of clinical characteristics have been little investigated. Therefore, we sought to assess potential prognostic factors for these patients in female Chinese patients and identify the treatment modalities they might benefit from, which offers implications for clinical practice.

Patients and methods: A total of 518 patients with four or more involved ALNs were retrospectively analyzed. Survival-curve analysis was performed with the Kaplan-Meier method, and Cox proportional hazard regression was applied to identify independent variables for disease-free survival (DFS) and overall survival (OS).

Results: The patients were divided into groups depending on the number of ALNs, with $38.22 \%$ having four to six positive ALNs and $61.78 \%$ having seven or more ALNs. Compared with the seven or more-positive ALN subgroup, patients with four to six positive ALNs tended to have smaller tumors and were more likely to undergo modified radical mastectomy rather than radical mastectomy (both $P<0.001$ ). Univariate analysis revealed that a fluorouracil/doxorubicin (epirubicin)/cyclophosphamide $(\mathrm{CA}[\mathrm{E}] \mathrm{F})$ regimen or a $\mathrm{CA}(\mathrm{E}) \mathrm{F}$ followed by docetaxel $(\mathrm{CA}[\mathrm{E}]$ $\mathrm{F}>\mathrm{T})$ regimen conferred significantly better DFS $(P=0.0075)$ and $\mathrm{OS}(P<0.0001)$ than those achieved from a cyclophosphamide/methotrexate/fluorouracil regimen, which was almost completely generated by the seven or more ALN subgroup ( $P=0.0088$ and $P=0.0001$, respectively). Postoperative radiotherapy was associated with better DFS $(P=0.0360)$, which was also generated by the seven or more ALN subgroup ( $P=0.0107)$. Subgroup analysis also clarified that the type of surgery conferred a modest effect on DFS in the seven or more ALN subgroup $(P=0.0305)$. Multivariate survival analysis revealed that ALN status (hazard ratio [HR] 2.00, 95\% confidence interval [CI] 1.31-3.05; $P=0.001$ ), tumor size (HR 1.48, 95\% CI 1.06-2.08; $P=0.022$ ), and type of surgery (HR $0.47,95 \%$ CI $0.30-0.74 ; P=0.001$ ) were independent prognostic factors for DFS. Meanwhile, ALN status (HR 2.96, 95\% CI 1.51-5.77; $P=0.002$ ), tumor size (HR 2.32, 95\% CI 1.38-3.89; $P=0.001)$, type of surgery $(\mathrm{HR}=0.39,95 \%$ CI $0.20-0.76$; $P=0.006$ ), and regimen of chemotherapy ( $\mathrm{HR}=0.64,95 \% \mathrm{CI} 0.50-0.85 ; P=0.002$ ) were identified as independent prognostic factors for OS.

Conclusion: Besides the classical prognostic factors and the improvement of prognosis achieved from the anthracycline-based or anthracycline-taxane combination chemotherapy compared to cyclophosphamide/methotrexate/fluorouracil chemotherapy, our findings showed benefits on DFS and OS for appropriate local treatments, including radiotherapy and sufficient ALN dissection for high-risk breast cancer patients with four or more ALNs involved, which suggests that much importance should also be attached to local treatment besides adjuvant systemic therapy.

Keywords: breast cancer, prognostic factor, adjuvant chemotherapy, adjuvant radiotherapy, surgery, high risk 


\section{Introduction}

Breast cancer is the most commonly diagnosed cancer and the second-leading cause of cancer-related death among women in the US, accounting for $29 \%$ of all new female cancer cases and $15 \%$ of all female cancer deaths. ${ }^{1}$ Although incidence rates vary, being lowest in Asia and Africa and highest in North America, a dramatic leap in the incidence rate has been observed in Asian countries over recent years, especially in the People's Republic of China. ${ }^{2}$ With the development of imaging techniques, the detection of early stage breast cancer has dramatically increased. ${ }^{3,4}$ However, there remains a significant number of breast cancer patients newly diagnosed with axillary lymph node (ALN) involvement.

As early as the 18th century, the German surgeon Lawrence Heister proposed that the involvement of ALNs could be a crucial factor for breast cancer metastasis. ${ }^{5}$ In 1891, Halsted established radical mastectomy including axillary dissection as a standard surgical approach, which has a good survival rate. ${ }^{6}$ Extended radical mastectomy and modified radical mastectomy were then developed and compared to this standard surgery. ${ }^{7-9}$ According to results from clinical trials, locoregional treatment including modified radical mastectomy and radiotherapy prevailed for decades because of similar effectiveness and less trauma. Nowadays, our modern understanding of the nature of breast cancer has revolutionized surgical treatment. A more conservative operation combining lumpectomy or mastectomy with sentinel LN biopsy (SLNB) has been proposed as an effective and less traumatic approach for axillary-negative cases. ${ }^{10}$ The SLNB technique provides reliable staging of ALN involvement and guides subsequent ALN dissection (ALND). The most recent trials have compared the effectiveness of SLNB and ALND, and indicate that axillary dissection can be safely avoided in patients with limited SLN involvement. ${ }^{11,12}$

Although the tendency of surgical patterns shifted dramatically over the years, ALND is still considered the standard procedure for patients with ALN involvement. ALN status and other factors are combined to evaluate the risk level of the breast cancer and guide chemotherapy. ${ }^{13}$ The status of ALNs is an important prognostic factor for breast cancer patients. Patients with four or more LNs involved can be independently categorized into the high-risk group without consideration of other clinical factors, according to the St Gallen consensus. For these patients, ALN status is considered a conclusively adverse prognostic factor. ${ }^{13}$ This group features a more aggressive clinical history and worse prognosis, despite intensive local and systemic treatments. However, the prognosis of these patients has been little investigated. Therefore, we sought to assess potential prognostic factors in Chinese women and identify treatment modalities, especially locoregional management, that they might benefit from, which offers implications for clinical practice.

\section{Patients and methods}

\section{Patients}

A total of 518 breast cancer patients with more than four positive ALNs were selected retrospectively from a large database of breast cancer patients who underwent surgery from February 1993 to January 2004 at Fudan University Shanghai Cancer Center (FUSCC). Eligibility criteria for this analysis included female sex, an initial diagnosis of unilateral primary breast cancer without distant metastases, more than four positive ALNs involved, at least 3 months of follow-up information on disease recurrence and death, and complete data on age, tumor size, number of ALNs involved, and surgery type. All eligible patients in the database were included. Patients with an initial diagnosis of distant metastases, fewer than four positive ALN involvement, or complicated with other invasive carcinoma were all excluded from this research.

Before surgery, each patient underwent a mandatory evaluation, including a complete physical examination, chest radioscopy, bilateral mammography, electrocardiography, ultrasonography of the breasts, axillary fossa, cervical parts, abdomen, pelvis, complete blood count, and routine biochemical tests, to diagnose the exact staging. All patients with level I-II ALND underwent modified radical mastectomy, and patients with level III ALND had radical mastectomy, followed by adjuvant therapies performed by physicians at FUSCC or in local hospitals according to current guidelines. None of the patients received trastuzumab therapy. Follow-up information, including adjuvant therapy, recurrence, and survival status, was collected by retrieval of medical records kept in the outpatient department and personal contact with the patient, including routine correspondence and telephone visits, which were carried out at FUSCC every 3 months during the first 2 years, every 6 months during the next 2 years, and once a year thereafter. In our database, follow-up was accomplished through the retrieval of medical records kept in the outpatient department, personal contact with the patient, and the assistance of the Shanghai Center for Disease Control and Prevention, requesting information regarding adjuvant treatment, tumor recurrence, and survival status. The presence of recurrence was collected by direct query, biopsy, or bone, abdomen, chest, pelvis, or skull scan. Whenever the 
tumor recurred, additional information, including the sites of recurrence and treatment therapy, was requested. All data were entered into a computerized database and verified to minimize errors in data entry.

Ethical appraisal from the Medical Ethics Committee at FUSCC was exempt for a retrospective study involving the collection or analysis of existing data in accordance with federal regulation 45 CFR 46. Therefore, written consent given by the patients was not needed for their hospitalization and follow-up information to be stored in the center database and research use. Furthermore, we took only anonymous data from the database, and the ethics committee waived the need for approval and consent.

\section{End-point definition and statistical analysis}

Disease-free survival (DFS) was defined as the time from surgery to the earliest occurrence of relapse (locoregional or distant) or death by any cause. Overall survival (OS) was defined as the time between surgery and death by any cause.

Survival curves were created using the Kaplan-Meier method, and were compared using the log-rank test. Multivariate Cox proportional hazard regression analysis was used to model the relationship between ALN status and survival, adjusted for age ( $\leq 35$ years, $>35$ and $\leq 50$ years, or $>50$ years), tumor size ( $\leq 2 \mathrm{~cm},>2 \mathrm{~cm}$ and $\leq 5 \mathrm{~cm}$, or $>5 \mathrm{~cm}$ ), hormone-receptor status (negative or positive), type of surgery (modified radical mastectomy or radical/ extended radical mastectomy), radiotherapy (no or yes), and chemotherapy regimen (cyclophosphamide/methotrexate/ fluorouracil (CMF), fluorouracil/doxorubicin (epirubicin)/ cyclophosphamide $[\mathrm{CA}\{\mathrm{E}\} \mathrm{F}]$, or $\mathrm{CA}[\mathrm{E}] \mathrm{F}$ followed by docetaxel $[\mathrm{CA}\{\mathrm{E}\} \mathrm{F}>\mathrm{T}]$ regimen). Hazard ratios (HRs) are presented with $95 \%$ confidence intervals (CIs). Results were considered statistically significant when the $P$-value was less than 0.05. All statistical analyses were performed with the Stata statistical software package (release 10.0; StataCorp LP, College Station, TX, USA).

\section{Results}

\section{General characteristics}

A total of 518 patients with four or more ALNs were analyzed. The mean age of the patients was 51.2 years, ranging from 28 to 84 years. The follow-up time varied from 0.25 to 10.67 years, the median follow-up duration was 2.12 years, and the mean follow-up duration was 3.04 years. According to the number of ALNs involved, patients were assigned to two subgroups: with four to six ALNs (38.22\%) or seven or more ALNs (61.78\%). Compared with the seven or more ALN subgroup, patients with four to six ALNs tended to have smaller tumors and were more likely to undergo a modified radical mastectomy rather than a radical/extended radical mastectomy (both $P<0.001$, Table 1).

\section{Survival analysis}

Univariate analysis showed that DFS and OS in patients with four to six ALNs were better than those for patients with seven or more ALNs $(P=0.0022$ and $P=0.0003$, respectively; Figure $1 \mathrm{~A}$ and $\mathrm{B})$. A similar prognostic benefit was seen in patients with smaller tumor size compared to patients with larger tumor size, which conferred better DFS and OS $(P=0.0047$ and $P=0.0009$, respectively; Figure $1 \mathrm{C}$ and $\mathrm{D})$.

As for biomarkers, both estrogen receptor- and progesterone receptor-positive status were associated with better DFS ( $P=0.0298$ and $P=0.0453$, respectively; Figure 2A and $\mathrm{C})$. Progesterone receptor status was also associated with OS ( $P=0.0107$, Figure 2D), whereas estrogen receptor status conferred an OS benefit with marginal significance $(P=0.0580$, Figure 2B).

When it came to therapies, there was no significant difference in DFS or OS between patients receiving a modified radical or radical/extended radical mastectomy $(P=0.2454$ and $P=0.6428$, respectively). To investigate further the effect of therapeutic approaches on patients with worse prognoses, we performed subgroup analysis, which clarified that the type of surgery conferred better DFS in the seven or more ALN subgroup ( $P=0.0305$, Figure $3 \mathrm{~A})$, and this analysis also showed an OS benefit of marginal significance $(P=0.0547$, Figure 3B). By contrast, radical/extended radical mastectomy failed to improve DFS $(P=0.6217)$ or $\operatorname{OS}(P=0.7761)$ for the four to six ALN subgroup (data not shown).

Postoperative radiotherapy was associated with better DFS ( $P=0.0360$, Figure 4A), but not with better OS $(P=0.2754$, data not shown). The significant difference for DFS was almost completely generated by the seven or more ALN subgroup ( $P=0.0107$, Figure 4B), whereas the four to six ALN subgroup did not reach statistical significance $(P=0.3344$, data not shown $)$.

In patients undergoing chemotherapy, anthracyclinebased or anthracycline-taxane combination chemotherapy conferred significantly better DFS $(P=0.0075)$ and OS $(P<0.0001)$ than those achieved from a $\mathrm{CMF}$ regimen (Figure 5A and B). Subgroup analysis also revealed that these differences were mainly generated by the seven or 
Table I Demographic and disease characteristics of eligible patients

\begin{tabular}{|c|c|c|c|}
\hline Characteristics & $\begin{array}{l}\text { Patients with 4-6 } \\
\text { ALNs }(n=198)\end{array}$ & $\begin{array}{l}\text { Patients with } \geq 7 \\
\text { ALNs }(n=320)\end{array}$ & $P$-value \\
\hline Age, years, n (\%) & & & 0.821 \\
\hline$\leq 35$ & $12(6.0)$ & $24(7.5)$ & \\
\hline$>35, \leq 50$ & $96(48.5)$ & $152(47.5)$ & \\
\hline$>50$ & $90(45.5)$ & $144(45)$ & \\
\hline Tumor size, $\mathrm{n}(\%)$ & & & $<0.0001$ \\
\hline $\mathrm{T} \leq 2 \mathrm{~cm}$ & $45(22.7)$ & 38 (1 I.9) & \\
\hline $2 \mathrm{~cm}<\mathrm{T} \leq 5 \mathrm{~cm}$ & $128(64.6)$ & $198(61.9)$ & \\
\hline $\mathrm{T}>5 \mathrm{~cm}$ & $25(12.6)$ & $84(26.3)$ & \\
\hline Estrogen-receptor status, n (\%) & & & 0.794 \\
\hline Positive & $103(52.0)$ & $169(52.8)$ & \\
\hline Negative & 75 (37.9) & $117(36.6)$ & \\
\hline Unknown & $20(10.1)$ & $34(10.6)$ & \\
\hline Progesterone-receptor status, n (\%) & & & 0.132 \\
\hline Positive & $99(50)$ & $|4|(44.1)$ & \\
\hline Negative & 75 (37.9) & $143(44.7)$ & \\
\hline Unknown & $24(12.1)$ & $36(11.2)$ & \\
\hline HER2 status, n (\%) & & & 0.105 \\
\hline Positive & $58(29.3)$ & II $4(35.6)$ & \\
\hline Negative & $119(60.1)$ & $169(52.8)$ & \\
\hline Unknown & $21(10.6)$ & $37(11.6)$ & \\
\hline Surgery type, n (\%) & & & $<0.0001$ \\
\hline Modified radical mastectomy & $76(38.4)$ & $70(21.9)$ & \\
\hline Radical/extended radical mastectomy & $122(61.6)$ & $250(78.1)$ & \\
\hline Chemotherapy regimen, $\mathrm{n}(\%)$ & & & 0.109 \\
\hline $\mathrm{CA}(\mathrm{E}) \mathrm{F} / \mathrm{CA}(\mathrm{E}) \mathrm{F}>\mathrm{T}$ & I I 8 (59.6) & $206(64.4)$ & \\
\hline CMF & $66(33.3)$ & $92(28.8)$ & \\
\hline No chemotherapy & $12(6.1)$ & II (3.4) & \\
\hline Unknown & $2(1.0)$ & II (3.4) & \\
\hline Radiotherapy, n (\%) & & & 0.04 \\
\hline Yes & $89(45.0)$ & $116(36.3)$ & \\
\hline No & $93(47.0)$ & $179(55.9)$ & \\
\hline Unknown & $16(8.0)$ & $25(7.8)$ & \\
\hline
\end{tabular}

Abbreviations: $\mathrm{CA}(\mathrm{E}) \mathrm{F}$, fluorouracil/doxorubicin (epirubicin)/cyclophosphamide; $\mathrm{CA}(\mathrm{E}) \mathrm{F}>\mathrm{T}, \mathrm{CA}(\mathrm{E}) \mathrm{F}$ followed by docetaxel; CMF, cyclophosphamide/methotrexate/ fluorouracil; ALNs, axillary lymph nodes.

more ALN subgroup $(P=0.0088$ and $P=0.0001$, respectively; Figure 5C and D).

\section{Multivariate analysis}

Finally, we performed a multivariate survival analysis, which included tumor size, number of LNs involved, hormone-receptor status, type of surgery, radiotherapy, and chemotherapy regimen. The analyses revealed that ALN status $(\mathrm{HR}=2.00,95 \% \mathrm{CI} 1.31-3.05 ; P=0.001)$, tumor size $(\mathrm{HR}=1.48,95 \% \mathrm{CI} 1.06-2.08 ; P=0.022)$, and type of surgery $(\mathrm{HR}=0.47,95 \% \mathrm{CI} 0.30-0.74 ; P=0.001)$ were independent prognostic factors for DFS. OS analysis revealed that ALN status $(\mathrm{HR}=2.96,95 \% \mathrm{CI} 1.51-5.77 ; P=0.002)$, tumor size $(\mathrm{HR}=2.32,95 \% \mathrm{CI} 1.38-3.89 ; P=0.001)$, type of surgery $(\mathrm{HR}=0.39$, 95\% CI $0.20-0.76 ; P=0.006)$, and chemotherapy regimen $(\mathrm{HR}=0.64,95 \%$ CI $0.50-0.85$;
$P=0.002)$ were identified as independent prognostic factors (Table 2).

\section{Discussion}

In recent years, numerous trials and studies focusing on early breast cancer have been carried out to optimize systemic adjuvant therapies, resulting in the minimization of surgical interventions for early stage breast cancer patients. In this context, our study was the first to focus on high-risk Chinese breast cancer patients with four or more ALNs involved, and aimed to explore the prognostic factors and more importantly to discuss the appropriate local treatments, especially the sufficient extent of ALND, that could improve the outcome for these high-risk patients.

Our results confirmed several classical prognostic factors that have been investigated previously, ${ }^{14,15}$ including 

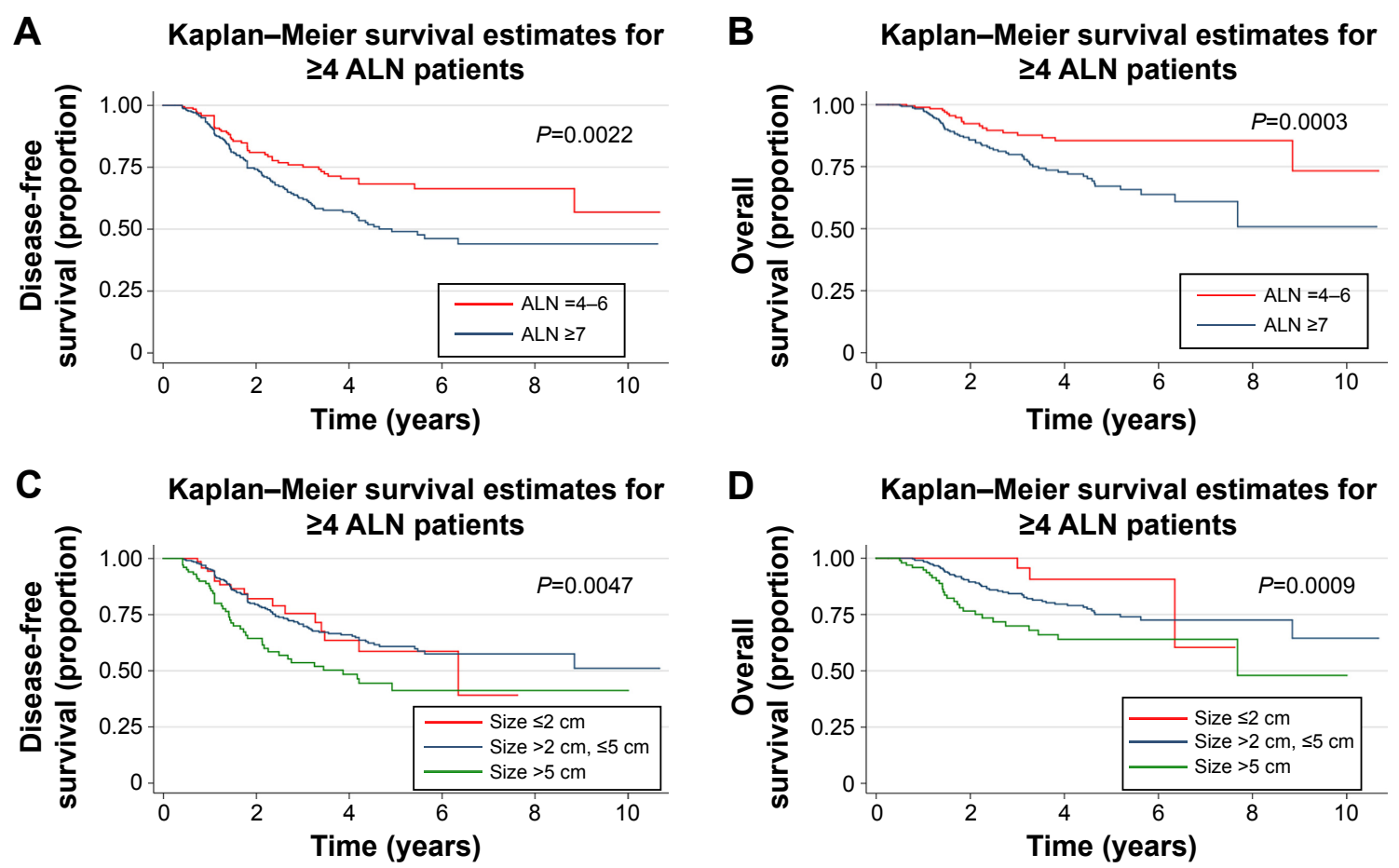

D Kaplan-Meier survival estimates for

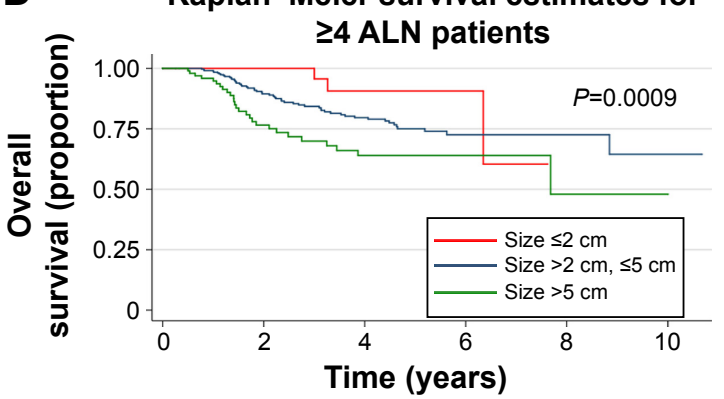

Figure I Survival analysis and subgroup analysis for patients with different axillary lymph node (ALN) status and tumor size.

Notes: Kaplan-Meier estimates of (A) disease-free survival and (B) overall survival for patients with different ALN status; Kaplan-Meier estimates of (C) disease-free survival and (D) overall survival for patients with different tumor sizes.
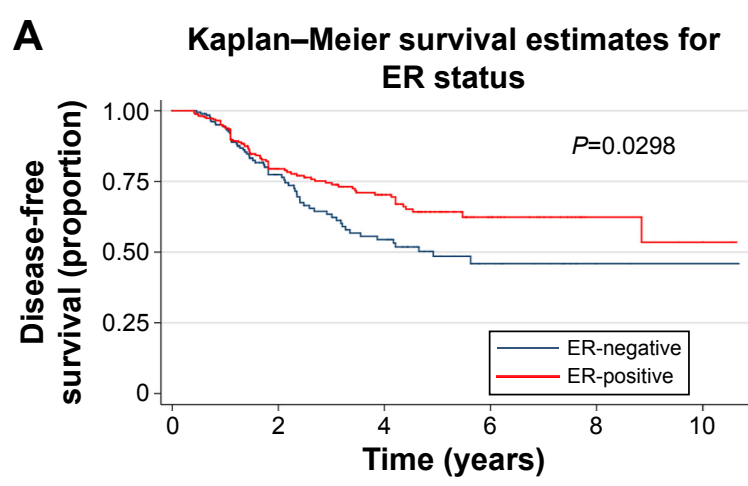

C

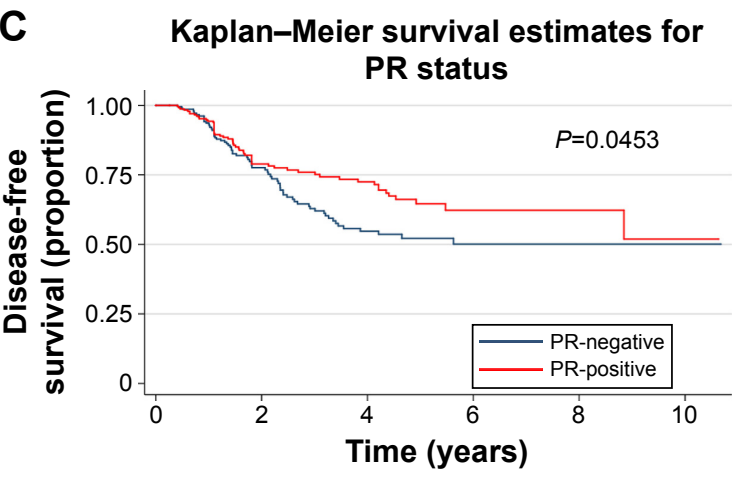

B Kaplan-Meier survival estimates for ER status

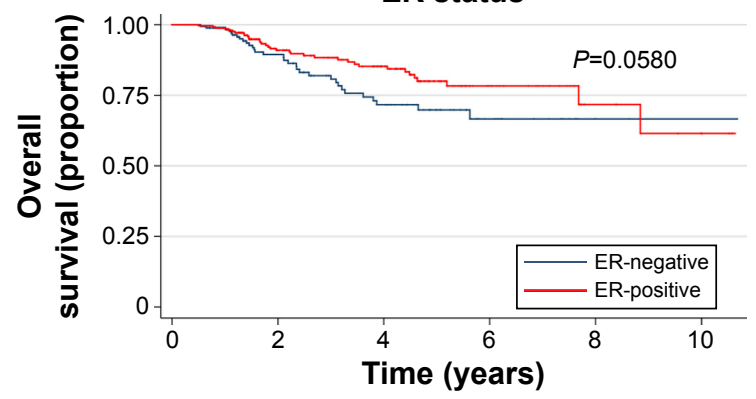

D Kaplan-Meier survival estimates for

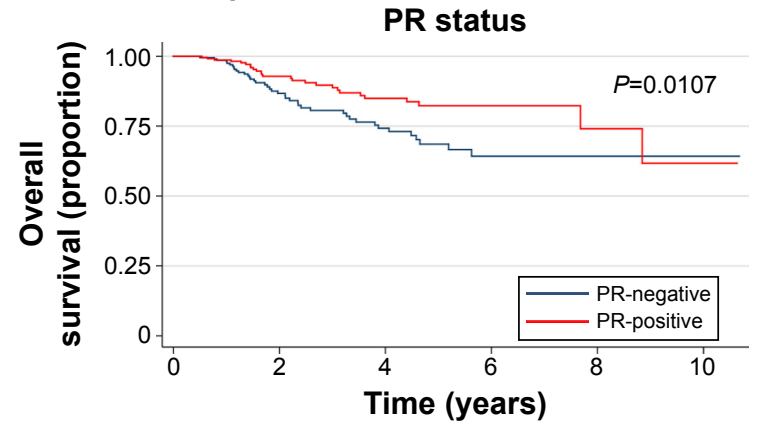

Figure 2 Survival analysis and subgroup analysis for patients with different hormone-receptor status.

Notes: Kaplan-Meier estimates of (A) disease-free survival and (B) overall survival for patients with different estrogen receptor (ER) status, and (C) disease-free survival and (D) overall survival for patients with different progesterone receptor (PR) status. 

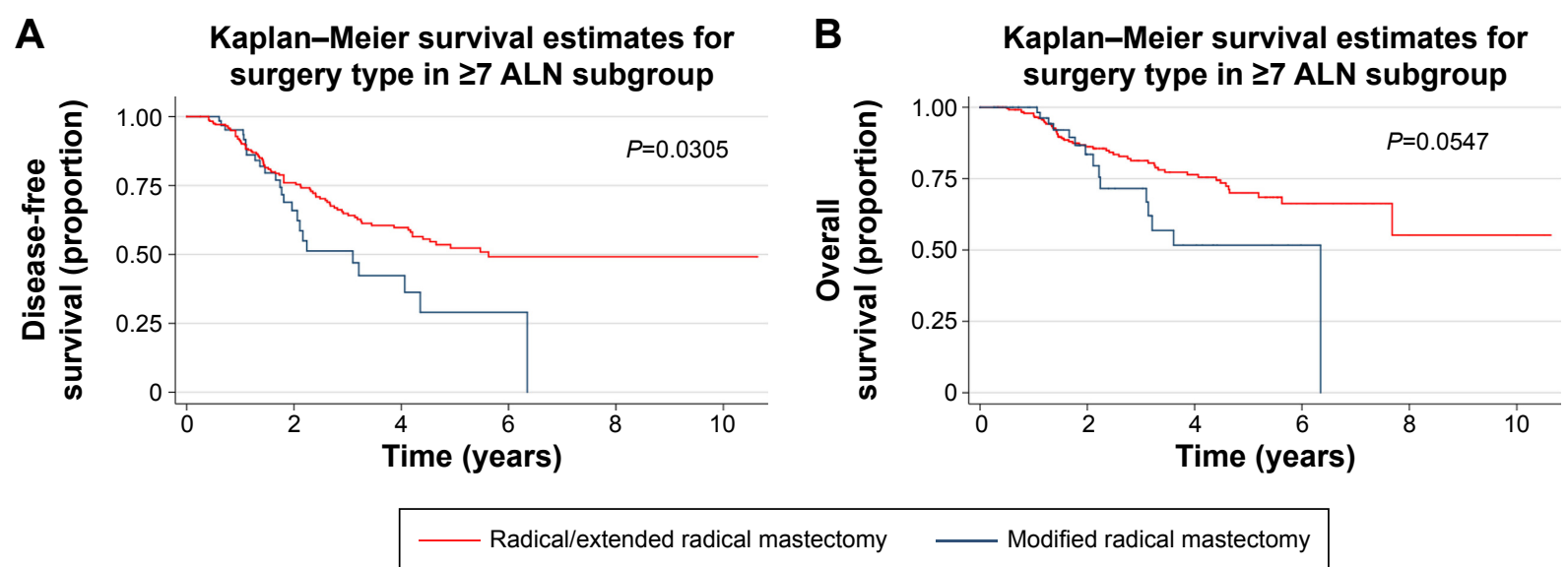

Figure 3 Survival analysis and subgroup analysis for patients undergoing different types of surgery.

Notes: Kaplan-Meier estimates of (A) disease-free survival and (B) overall survival for patients with seven or more axillary lymph nodes (ALNs).

tumor size, LN involvement, and hormone-receptor status. These factors conferred a similar prognosis for high-risk patients with four or more ALNs involved, as has been seen in previous studies focusing on breast cancer with fewer than four ALNs.

Based on our data, the analysis of chemotherapy regimens indicated that anthracycline-based or anthracycline-taxane combination chemotherapy conferred better DFS and OS than those achieved from a CMF regimen, which corroborated results from several trials of anthracycline-containing regimens that found that they were superior to a CMF regimen for node-positive patients. ${ }^{16-18}$ According to these solid results, anthracycline-containing regimens are qualified as appropriate chemotherapy regimens for node-positive patients in the National Comprehensive Cancer Network guidelines. ${ }^{19}$

Meanwhile, our data showed that adjuvant radiotherapy was associated with both better DFS and OS. As a part of local treatment for high-risk breast cancer, radiotherapy eradicated local residual and metastatic lesions, which usually existed both locally and systemically in postsurgery patients with four or more ALNs involved. Even in early stage breast cancer, the omission of surgical intervention on the axilla must be based on successful radiotherapy of the whole breast. ${ }^{12}$ The NASBP-04 trial and a French trial revealed no significant difference in DFS or OS between ALND and axillary radiotherapy. ${ }^{20,21}$ Recent results from the EORTC 10981-22023 AMAROS trial also confirmed the similar effectiveness of both treatments, which revealed that both ALND and axillary radiotherapy after a positive SLNB provided excellent and comparable regional control for patients with $\mathrm{T} 1-\mathrm{T} 2$ primary breast cancer. ${ }^{22} \mathrm{~A}$ meta-analysis from that study revealed that radiotherapy also reduced both the recurrence and mortality rates in patients with one to three positive LNs, even when systemic therapy was given. ${ }^{23}$ Our
A

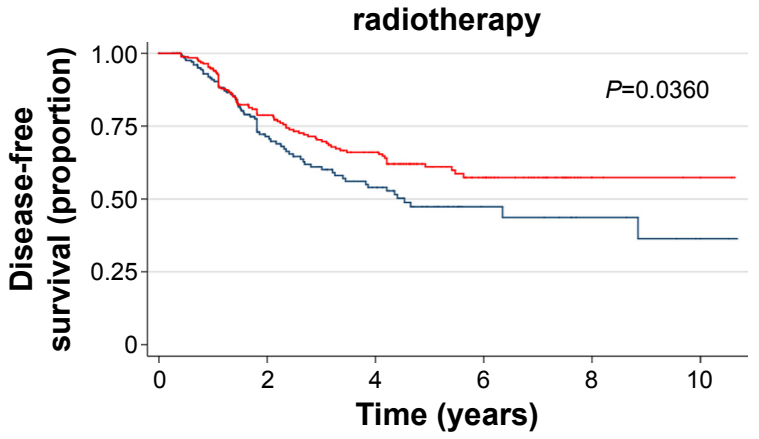

B
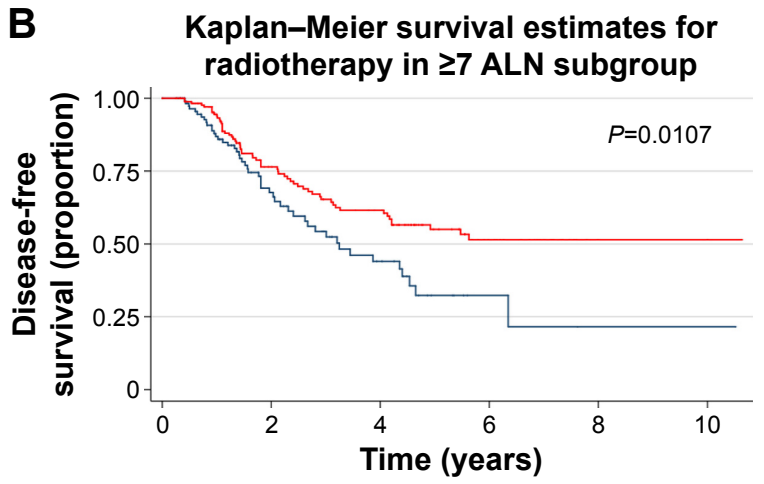

Radiotherapy — No radiotherapy

Figure 4 Survival analysis and subgroup analysis for patients with or without radiotherapy.

Notes: Kaplan-Meier estimates of (A) disease-free survival for all patients and (B) disease-free survival for patients with seven or more ALNs involved with or without radiotherapy. 
A

Kaplan-Meier survival estimates for chemotherapies

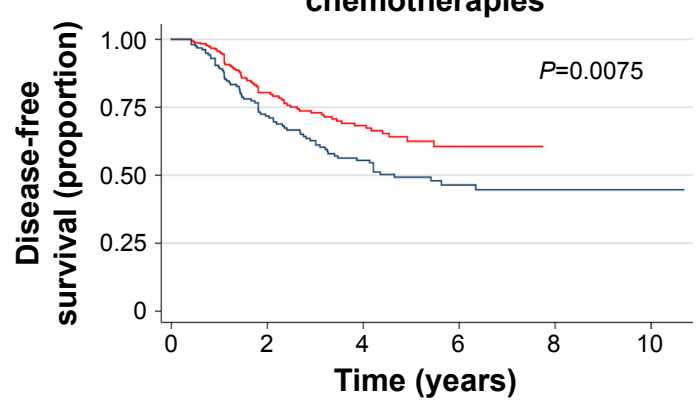

C Kaplan-Meier survival estimates for chemotherapies in $\geq 7$ ALN subgroup

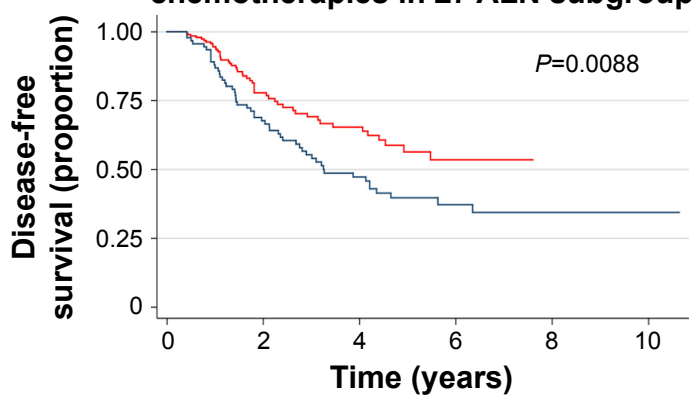

B Kaplan-Meier survival estimates for chemotherapies

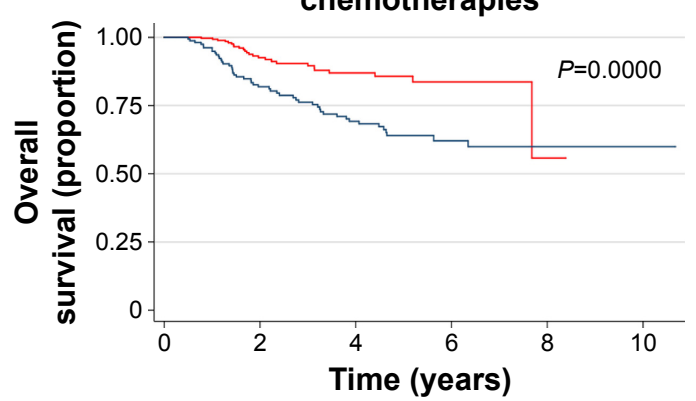

D Kaplan-Meier survival estimates for chemotherapies in $\geq 7$ ALN subgroup

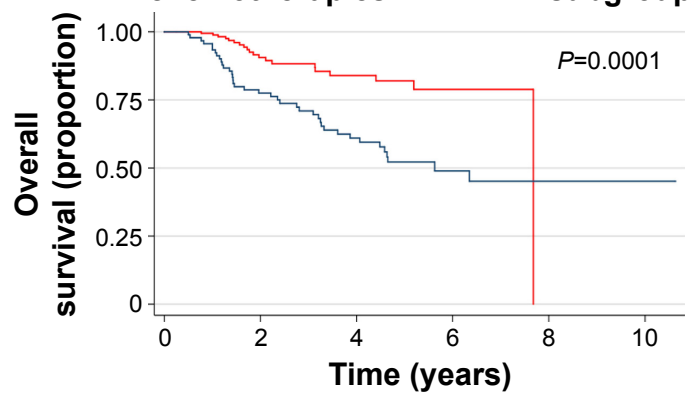

$$
\longrightarrow \mathrm{CA}(\mathrm{E}) \mathrm{F} / \mathrm{CA}(\mathrm{E}) \mathrm{F}>\mathrm{T} \quad \mathrm{CMF}
$$

Figure 5 Survival analysis and subgroup analysis for patients receiving different chemotherapy regimens.

Notes: Kaplan-Meier estimates of (A) disease-free survival and (B) overall survival for all patients, (C) ALN disease-free survival and (D) overall survival for patients with seven or more ALNs involved receiving different chemotherapy regimens.

Abbreviations: $\mathrm{CA}(\mathrm{E}) \mathrm{F}$, fluorouracil/doxorubicin (epirubicin)/cyclophosphamide; $\mathrm{CA}(\mathrm{E}) \mathrm{F}>\mathrm{T}, \mathrm{CA}(\mathrm{E}) \mathrm{F}$ followed by docetaxel; CMF, cyclophosphamide/methotrexate/ fluorouracil.

results validated the importance of radiotherapy by showing a statistically significant difference for improving DFS and OS. Adjuvant radiotherapy has the advantage of high effectiveness on regional control for early stage patients, and also shows survival benefits for high-risk patients with four or more ALNs involved.

Besides showing that more aggressive adjuvant chemotherapy improved the prognosis for high-risk patients, our analysis on radiotherapy also revealed that local treatment improved the prognosis of high-risk patients. As such, another aim of our study was to analyze the efficiency of local surgical treatments for high-risk patients. Our results revealed that more aggressive surgery, including the dissection of level III ALNs, was associated with better DFS and OS for high-risk patients, and this benefit was more apparent in patients with seven or more ALNs involved. Consequently, ALND should be performed to a sufficient extent, and these findings may help guide decision making during surgical intervention. The

Table 2 Multivariate Cox proportional hazard regression analyses for DFS and OS in eligible patients

\begin{tabular}{|c|c|c|c|c|c|c|}
\hline \multirow[t]{2}{*}{ Variable } & \multicolumn{3}{|l|}{ DFS } & \multicolumn{3}{|l|}{ OS } \\
\hline & HR & $95 \% \mathrm{Cl}$ & $P$-value & HR & $95 \% \mathrm{Cl}$ & $P$-value \\
\hline Age & 0.75 & $0.55-1.01$ & 0.058 & 0.80 & $0.5 \mathrm{I}-\mathrm{I} .24$ & 0.314 \\
\hline Number of ALNs involved & 2.00 & $1.31-3.05$ & 0.001 & 2.96 & $\mid .5 \mathrm{I}-5.77$ & 0.002 \\
\hline Tumor size & $\mathrm{I} .48$ & $1.06-2.08$ & 0.022 & 2.32 & I.38-3.89 & 0.001 \\
\hline Hormone-receptor status & 0.88 & $0.58-1.32$ & 0.529 & 0.82 & $0.45-1.46$ & 0.495 \\
\hline Type of surgery & 0.47 & $0.30-0.74$ & 0.001 & 0.39 & $0.20-0.76$ & 0.006 \\
\hline Radiotherapy & 0.72 & $0.49-1.05$ & 0.086 & 0.89 & $0.5 I-1.55$ & 0.687 \\
\hline Chemotherapy regimen & 0.87 & $0.72-1.05$ & 0.151 & 0.64 & $0.50-0.85$ & 0.002 \\
\hline
\end{tabular}

Abbreviations: DFS, disease-free survival; OS, overall survival; HR, hazard ratio; $\mathrm{Cl}$, confidence interval; ALNs, axillary lymph nodes. 
surgical range of ALND has been greatly reduced, due to the development of diagnostic techniques and new concepts in local treatment. The development of improved diagnostics has facilitated effective early diagnosis before the ALNs get involved, and SLNB has routinely been performed for further surgical decision making. According to the results of the IBCSG 23-01 and ACOSOG Z0011 trials, the tendency to omit ALND in patients with micrometastases in one or more sentinel LNs has been accepted by an increasing number of surgeons and may become the standard of practice in the near future. ${ }^{11,12}$ Furthermore, an even more aggressive concept has been proposed and is being validated in a current trial, which will explore the possibility of omitting SLNB in patients with ultrasound-negative axilla. ${ }^{24}$ Although surgical treatment of the axilla for early stage patients has been greatly optimized by these trials, few studies have focused on axillary treatment for high-risk patients with four or more ALNs involved, and no standard procedure or consensus has been developed. As an important procedure for local treatment, surgical axillary treatment should be emphasized for late-stage patients because of the high metastasis and mortality rate. Specific and precise guidelines for high-risk patients should also be developed for standardizing treatment in the axilla.

Surgery of the axilla is necessary for the eradication of metastatic LNs. However, the current challenge is in deciding whether to indiscriminately minimize the extent of surgery for all patients in compliance with contemporary guidelines, followed by radiotherapy and systemic therapies to treat possible residual lesions and metastases. The data from our analyses may provide some clues. Survival analysis revealed that radical/extended radical mastectomy, including level III ALND, played a crucial role in improving DFS and OS for patients with four or more ALNs involved. Subgroup analysis showed the importance of level III ALND for the seven or more involved ALN subgroup as well, which presented better DFS and a marginally significant improvement in OS. However, radical/extended radical mastectomy did not have an advantage for patients with four to six ALNs. These data might indicate that surgery, including axillary dissection, could improve the prognosis for patients with seven or more ALNs involved. Compared to radiotherapy, aggressive surgery also had a comparable effect on improving the prognosis of extremely high-risk patients. Still, surgical interventions are different from radiotherapy and chemotherapy, which seek to eradicate most tumor cells and reduce the tumor burden to a minimal level, thus facilitating therapies targeting residual or metastatic lesions. For high-risk patients with four or more ALN metastases, ALND is a crucial procedure that can only be performed by a surgeon, and the clearance rate of ALNs depends on the surgeon's decision to proceed and attentiveness during the surgery. Residual LN metastases could result in postoperative recurrence despite the use of effective therapies. The National Comprehensive Cancer Network guidelines for breast cancer also agree that ALND should be extended to include level III cases if gross disease is apparent in level II nodes. ${ }^{19}$ According to our results, we suggest the use of ALND be extended to level III clearance in these high-risk patients. A mandatory level III axillary clearance might be recommended as a standard surgical procedure for maximum survival improvement, especially for patients with seven or more ALNs involved.

A potential limitation of this analysis is that the immunohistochemistry diagnosis of HER2 varied due to the lack of consistent and standard criteria, and none of the patients received Herceptin therapy during the analysis period. It was difficult to analyze the prognostic effect of HER2 without a standardized protocol and quality-control measures. Furthermore, the follow-up duration was quite short, and a longer follow-up period would allow for more accurate and reliable results. Because of these limitations and the retrospective nature of this study, our results should be used for hypothesis generation only and need to be confirmed in subsequent large prospective studies. Additionally, the anthracyclinebased and anthracycline-taxane combination chemotherapy treatments were combined into a single group. Because few patients received anthracycline-taxane combination chemotherapy during the analysis period, the different effects of anthracycline-based and anthracycline-taxane combination chemotherapy could not be analyzed accurately.

In conclusion, our data show that aggressive chemotherapy regimens and appropriate local treatments, including radiotherapy and sufficient ALND extent, conferred survival benefits for high-risk breast cancer patients with four or more ALNs involved. Level III ALND conferred significantly better survival for patients with seven or more ALNs involved, and suggests that local treatment, served as crucial initial therapy, could help these extremely highrisk patients in improving their survival rate. Although the general tendency in therapy for breast cancer is to minimize surgical intervention and even to omit ALND for early stage breast cancer patients, ALND to a sufficient extent may be important for improving the survival rate and prognosis of high-risk patients in clinical practice.

\section{Disclosure}

The authors report no conflicts of interest in this work. 


\section{References}

1. Siegel R, Ma J, Zou Z, Jemal A. Cancer statistics, 2014. CA Cancer J Clin. 2014;64(1):9-29.

2. [No authors listed]. Cancer incidence in five continents. Volume IX. IARC Sci Publ. 2008;(160):1-837.

3. Bluekens AM, Holland R, Karssemeijer N, Broeders MJ, den Heeten GJ. Comparison of digital screening mammography and screen-film mammography in the early detection of clinically relevant cancers: a multicenter study. Radiology. 2012;265(3):707-714.

4. Fracheboud J, Otto SJ, van Dijck JA, Broeders MJ, Verbeek AL, de Koning HJ. Decreased rates of advanced breast cancer due to mammography screening in the Netherlands. Br J Cancer. 2004;91(5):861-867.

5. Meyer KK, Beck WC. Mastectomy performed by Lawrence Heister in the eighteenth century. Surg Gynecol Obstet. 1984;159(4):391-394.

6. Halsted WS. I. The results of operations for the cure of cancer of the breast performed at the Johns Hopkins Hospital from June, 1889, to January, 1894. Ann Surg. 1894;20(5):497-555.

7. Lacour J, Le M, Caceres E, Koszarowski T, Veronesi U, Hill C. Radical mastectomy versus radical mastectomy plus internal mammary dissection. Ten year results of an international cooperative trial in breast cancer. Cancer. 1983;51(10):1941-1943.

8. Maddox WA, Carpenter JT Jr, Laws HL, et al. A randomized prospective trial of radical (Halsted) mastectomy versus modified radical mastectomy in 311 breast cancer patients. Ann Surg. 1983;198(2):207-212.

9. Bijker N, Rutgers EJ, Peterse JL, et al. Low risk of locoregional recurrence of primary breast carcinoma after treatment with a modification of the Halsted radical mastectomy and selective use of radiotherapy. Cancer. 1999;85(8):1773-1781.

10. Veronesi U, Paganelli G, Galimberti V, et al. Sentinel-node biopsy to avoid axillary dissection in breast cancer with clinically negative lymph-nodes. Lancet. 1997;349(9069):1864-1867.

11. Galimberti V, Cole BF, Zurrida S, et al. Axillary dissection versus no axillary dissection in patients with sentinel-node micrometastases (IBCSG 23-01): a phase 3 randomised controlled trial. Lancet Oncol. 2013;14(4):297-305.

12. Giuliano AE, Hunt KK, Ballman KV, et al. Axillary dissection vs no axillary dissection in women with invasive breast cancer and sentinel node metastasis: a randomized clinical trial. JAMA. 2011;305(6):569-575.

13. Goldhirsch A, Glick JH, Gelber RD, et al. Meeting highlights: international expert consensus on the primary therapy of early breast cancer 2005. Ann Oncol. 2005;16(10):1569-1583.
14. Bundred NJ. Prognostic and predictive factors in breast cancer. Cancer Treat Rev. 2001;27(3):137-142.

15. Rampaul RS, Pinder SE, Elston CW, Ellis IO. Prognostic and predictive factors in primary breast cancer and their role in patient management: the Nottingham Breast Team. Eur J Surg Oncol. 2001;27(3):229-238.

16. [No authors listed]. Polychemotherapy for early breast cancer: an overview of the randomised trials. Early Breast Cancer Trialists' Collaborative Group. Lancet. 1998;352(9132):930-942.

17. Levine MN, Pritchard KI, Bramwell VH, Shepherd LE, Tu D, Paul N. Randomized trial comparing cyclophosphamide, epirubicin, and fluorouracil with cyclophosphamide, methotrexate, and fluorouracil in premenopausal women with node-positive breast cancer: update of National Cancer Institute of Canada Clinical Trials Group Trial MA5. J Clin Oncol. 2005;23(22):5166-5170.

18. French Adjuvant Study Group. Benefit of a high-dose epirubicin regimen in adjuvant chemotherapy for node-positive breast cancer patients with poor prognostic factors: 5-year follow-up results of French Adjuvant Study Group 05 randomized trial. J Clin Oncol. 2001;19(3):602-611.

19. Gradishar WJ, Anderson BO, Blair SL, et al. Breast cancer version 3. J Natl Compr Canc Netw. 2014;12(4):542-590.

20. Fisher B, Jeong JH, Anderson S, Bryant J, Fisher ER, Wolmark N. Twenty-five-year follow-up of a randomized trial comparing radical mastectomy, total mastectomy, and total mastectomy followed by irradiation. N Engl J Med. 2002;347(8):567-575.

21. Louis-Sylvestre C, Clough K, Asselain B, et al. Axillary treatment in conservative management of operable breast cancer: dissection or radiotherapy? Results of a randomized study with 15 years of follow-up. J Clin Oncol. 2004;22(1):97-101.

22. Donker M, van Tienhoven G, Straver ME, et al. Radiotherapy or surgery of the axilla after a positive sentinel node in breast cancer (EORTC 10981-22023 AMAROS): a randomised, multicentre, open-label, phase 3 non-inferiority trial. Lancet Oncol. 2014;15(12):1303-1310.

23. McGale P, Taylor C, Correa C, et al. Effect of radiotherapy after mastectomy and axillary surgery on 10-year recurrence and 20-year breast cancer mortality: meta-analysis of individual patient data for 8135 women in 22 randomised trials. Lancet. 2014;383(9935):2127-2135.

24. Gentilini O, Veronesi U. Abandoning sentinel lymph node biopsy in early breast cancer? A new trial in progress at the European Institute of Oncology of Milan (SOUND: Sentinel node vs Observation after axillary UltraSouND). Breast. 2012;21(5):678-681.
OncoTargets and Therapy

\section{Publish your work in this journal}

OncoTargets and Therapy is an international, peer-reviewed, open access journal focusing on the pathological basis of all cancers, potential targets for therapy and treatment protocols employed to improve the management of cancer patients. The journal also focuses on the impact of management programs and new therapeutic agents and protocols on

\section{Dovepress}

patient perspectives such as quality of life, adherence and satisfaction. The manuscript management system is completely online and includes a very quick and fair peer-review system, which is all easy to use. Visit http://www.dovepress.com/testimonials.php to read real quotes from published authors. 